\title{
Immortal time bias does not invalidate the association of self-monitoring of blood glucose with better survival of patients with type 2 diabetes in the epidemiological study ROSSO (Retrolective Study: Self-monitoring of Blood Glucose and Outcome in Patients with Type 2 Diabetes)
}

\author{
B. Schneider • S. Martin • W. A. Scherbaum • \\ L. Heinemann • V. Lodwig • H. Kolb
}

Received: 6 October 2010 / Accepted: 7 October 2010 /Published online: 20 November 2010

(C) Springer-Verlag 2010

Keywords Bias $\cdot$ Cohort studies $\cdot$ Mortality

Self-monitoring of blood glucose $\cdot$ Type 2 diabetes

\begin{abstract}
Abbreviations
ROSSO Retrolective Study: Self-monitoring of Blood Glucose and Outcome in Patients with Type 2 Diabetes

SMBG Self-monitoring of blood glucose
\end{abstract}

To the Editor:

We read Hoffmann and Andersohn's article that was published recently in Diabetologia [1] with interest. They conclude that our retrospective study of self-monitoring of blood glucose (SMBG) and outcome in patients with type 2 diabetes (Retrolective Study: Self-monitoring of Blood Glucose and Outcome in Patients with Type 2 Diabetes; ROSSO) was severely flawed. They conclude that, contrary to what we have published [2], SMBG is not associated

B. Schneider

Institute of Biometry, Hanover Medical University,

Hanover, Germany

S. Martin $(\bowtie)$

West-German Centre of Diabetes and Health,

Sana Clinics Düsseldorf,

Gräulinger Str. 120,

40625 Düsseldorf, Germany

e-mail: s.martin@sana-duesseldorf.de

W. A. Scherbaum

Department of Endocrinology, Diabetes and Rheumatology,

University Hospital Düsseldorf,

Düsseldorf, Germany with a $51 \%$ reduction of all-cause mortality, but rather, that SMBG is associated with an almost doubled risk (195\%).

Hoffmann and Andersohn claim that our analysis ignored immortal time bias. This type of bias may indeed strongly affect the outcome of epidemiological studies. They refer to work by Suissa [3], who suggested using Cox proportional hazards modelling with time-dependent exposures to avoid immortal time bias. Hoffmann and Andersohn were apparently not aware that we published such an analysis 3 years ago [4]. The time-dependence of SMBG and treatment with insulin and oral glucoselowering drugs was modelled by time functions of followup years with value 1 if in a year the manoeuvre (SMBG, therapy with insulin, therapy with oral glucose-lowering drug) was performed, and 0 otherwise. For the hazard of non-fatal or fatal endpoints the estimated coefficient $\exp (\mathrm{B})$ for the time function of follow-up years with SMBG is 0.037 (95\% CI $0.009-0.159)$. This suggests a statistically significant benefit of SMBG use in decreasing the hazard for

L. Heinemann

Profil Institute for Metabolic Research,

Neuss, Germany

V. Lodwig

Roche Diagnostics,

Mannheim, Germany

H. Kolb

Immunobiology Research Group, Institute of Molecular Medicine,

University of Düsseldorf,

Düsseldorf, Germany 
non-fatal or fatal events. In summary, we have performed the proper analysis to exclude an impact of immortal time bias and published the article in a journal with a peer-review system, accessible by PubMed. Thus Hoffmann and Andersohn's critique of ROSSO lacks substance.

When applying statistical methods for the evaluation of outcome in patients with type 2 diabetes, it is of paramount importance to be aware of the natural course of the disease and of the biochemical processes involved, otherwise statistical tests may lead to wrong conclusions. First, we observed in ROSSO that survival of patients was significantly better in those younger at diagnosis [5], and that patients performing SMBG were significantly younger than those who did not [2]. This has a major impact on immortal time bias, but was not considered by Hoffmann and Andersohn when assessing immortal time bias. Second, disease progression was not linear; rather, we found that SMBG was introduced in response to deterioration of metabolic control [6]. Hence, disease quality was much more benign prior to the onset of SMBG. Again, this has a major bearing on immortal time bias, but again this was not considered by Hoffmann and Andersohn. Third, the calculation of immortal time bias by Hoffmann and Andersohn is based on the assumption that the influence of SMBG on the endpoints depends strictly on the duration of SMBG. This is not the case, because SMBG is a diagnostic tool and in itself has no direct influence on the endpoints. Such an influence will only be achieved if the patient and/or the treating physician use the information derived from SMBG for modification of glucose-lowering treatment and lifestyle. We reported [4] that the initiation of SMBG was associated with intensified glucose-lowering therapy (e.g. introduction of oral glucose-lowering drugs or of insulin). Patients who did not use SMBG changed therapy significantly less often, even if a rise of fasting blood glucose levels indicated insufficient blood glucose control. Because of the indirect and non-linear relationship between the use of SMBG and diabetes treatment, the adjustment of observational person-years by subtracting and adding immortal time will not reduce bias but produce additional bias, and is not applicable to the ROSSO study.

In studies of multiple sclerosis, the calculation of immortal time bias without considering a non-linear disease process recently led to the conclusion that therapy with interferon- $\beta$ speeds up disease progression, which is in conflict with evidence from randomised controlled trials [7]. Unfortunately, there is no randomised controlled trial of patient mortality vs the use of SMBG in patients with type 2 diabetes. Hoffmann and Andersohn's statement that the results of ROSSO 'conflict with findings from randomised controlled trials' is therefore unfounded and incorrect.

The only true bias in the paper by Hoffmann and Andersohn appears to be the statement that 'the ROSSO study is clearly "too good to be true". We are surprised that the authors included such an emotive statement in a scientific article. Indeed, simple lifestyle changes caused remission for at least 6 years in more than $50 \%$ of the individuals with early type 2 diabetes who enrolled in the Malmö feasibility study [8]. Similarly, modest changes of lifestyle also prevented the progression to type 2 diabetes during the initial study period by more than $50 \%$ in the Finnish Diabetes Prevention Trial and the Diabetes Prevention Program [9, 10]. Too good to be true?

We are happy to share further unpublished analyses with Hoffmann and Andersohn if they wish. The ROSSO dataset is special in that it documents the natural course of type 2 diabetes from diagnosis in a large cohort. Direct collaborative interactions are welcome.

Duality of interest S. Martin and H. Kolb have received lecture fees for speaking at the invitation of Roche Diagnostics; H. Kolb has also received lecture fees from Bayer. V. Lodwig is employed by the company that sponsored the study (Roche Diagnostics). L. Heinemann holds shares in the Profil Institute for Metabolic Research, which received grant support for the ROSSO study. None of the other authors have any potential conflicts of interest relevant to this article.

\section{References}

1. Hoffmann F, Andersohn F (2010) Immortal time bias and survival in patients who self-monitor blood glucose in the retrolective study: self-monitoring of blood glucose and outcome in patients with type 2 diabetes (ROSSO). Diabetologia. doi:10.1007/s00125-010-1909-3

2. Martin S, Schneider B, Heinemann L et al (2006) Self-monitoring of blood glucose in type 2 diabetes and long-term outcome: an epidemiological cohort study. Diabetologia 49:271-278

3. Suissa S (2008) Immortal time bias in pharmaco-epidemiology. Am J Epidemiol 167:492-499

4. Schneider B, Martin S, Heinemann L, Lodwig V, Kolb H (2007) Interrelations between diabetes therapy, self-monitoring of blood glucose, blood glucose and non-fatal or fatal endpoints in patients with type 2 diabetes/results of a longitudinal cohort study (ROSSO 5). Arzneimittelforschung 57:762-769

5. Kolb H, Schneider S, Heinemann L et al (2008) Type 2 diabetes phenotype and progression is significantly different if diagnosed before vs after 56 years of age. J Diabetes Sci Technol 2:82-90

6. Kolb H, Schneider B, Heinemann L, Lodwig V, Scherbaum WA, Martin S (2007) Altered disease course after initiation of selfmonitoring of blood glucose in noninsulin-treated type 2 diabetes (ROSSO 3). J Diabetes Sci Technol 1:487-495

7. Trojano M, Pellegrini F (2008) Reply to Renoux C, Suissa S: Immortal time bias in the study of effectiveness of interferon- $\beta$ in multiple sclerosis. Ann Neurology 64:110

8. Eriksson KF, Lindgarde F (1991) Prevention of type 2 (noninsulin-dependent) diabetes mellitus by diet and physical exercise. The 6-year Malmö feasibility study. Diabetologia 34:891-898

9. Tuomilehto J, Lindstrom J, Eriksson JG et al (2001) Prevention of type 2 diabetes mellitus by changes in lifestyle among subjects with impaired glucose tolerance. N Engl J Med 344:1343-1350

10. Diabetes Prevention Program Research Group (2002) Reduction in the incidence of type 2 diabetes with lifestyle intervention or metformin. N Engl J Med 346:393-403 